\section{Postinfectious brainstem encephalitis associated with SARS-CoV-2}

\section{INTRODUCTION}

Immune-mediated neurological sequelae following infection with severe acute respiratory syndrome coronavirus 2 (SARS-CoV-2) are becoming increasingly evident, and can affect both the peripheral and central nervous system. ${ }^{12}$ We report a postinfectious brainstem syndrome in a patient with COVID-19 who presented with generalised myoclonus, ocular flutter with convergence spasm and acquired hyperekplexia. Clinical improvement was seen following corticosteroids, highlighting this as a possible treatment in patients where a post-COVID-19 autoimmune encephalitis is suspected.

\section{CASE PRESENTATION}

A 65-year-old woman presented with a 1 -week history of widespread involuntary movements, diplopia and cognitive decline having experienced fever, cough and myalgias the week before.

She had a medical history of presumed Alzheimer's disease, osteoarthritis and gastro-oesophageal reflex disease. The diagnosis of Alzheimer's disease had been made 3 years earlier on the basis of a 12-month history of amnestic symptoms including repetitive questioning and navigational difficulties. At her functional baseline, she could mobilise unaided, leave the house independently and wash without assistance. She was fluent in English, but her native language was Arabic. MiniMental State Examination score 8 months prior to presentation was $20 / 24$. Regular medications included a rivastigmine patch $4.6 \mathrm{mg} / 24$ hours, omeprazole and solifenacin.

Collateral information from her son reported that 2 weeks prior to presentation she had developed non-productive cough, fever and myalgias. She had also experienced 2 days of diarrhoea which spontaneously resolved. Her son suffered from similar symptoms and both members of the household self-isolated for suspected COVID-19.

Although these symptoms gradually improved, 7 days later she developed involuntary movements, initially in the right hand and upper arm. Over the next 48 hours, these movements became unremitting and generalised, involving the face and all four limbs. She began to only speak in her native Arabic, before developing progressive speaking difficulties and increasing confusion. Over this time, she also described worsening vision with difficulty reading, struggling to focus and intermittent double vision. In the 24 hours prior to admission, she experienced wellformed visual hallucinations of people in the house and seeing objects flying around the room, prompting attendance to hospital.

The most prominent feature on neurological examination was widespread, stimulus-sensitive myoclonus involving the tongue and all four limbs, associated with hyperekplexia without habituation to tactile, visual and auditory stimuli (see online supplementary video 1 ). There was a full range of extraocular eye movements with associated ocular-facial synkinesis. Saccadic eye movements were interrupted by ocular flutter and prominent convergence spasm with miosis on visual fixation (see online supplementary video 1 ). She was alert and orientated to place but not time (day/year) and was only able to follow single stage commands. She had a non-fluent aphasia and difficulty repeating sentences. Perseveration, echopraxia and marked mirror movements were evident on motor testing, but constructional apraxia was absent. Allowing for persistent myoclonus, the remainder of the neurological examination was normal.

Combined throat and high nasal swab reverse-transcriptase polymerasechain-reaction testing was positive for SARS-CoV-2. Chest X-ray demonstrated bilateral peripheral pulmonary infiltrates in the mid and lower zones consistent with COVID-19. Complete blood examination including lymphocyte count was normal. C-reactive protein was raised at $21 \mathrm{mg} / \mathrm{L}$. D-dimer was $1800 \mu \mathrm{g} / \mathrm{L}$ (normal <500).

MRI of the brain with contrast, including three-dimensional volumetric fluid-attenuated inversion recovery (FLAIR) and diffusion weighted imaging, was normal. Cerebrospinal fluid (CSF) examination showed a white cell count of $0.001 \times 10^{9} / \mathrm{L}$ (normal $<0.005 \times 10^{9} / \mathrm{L}$ ), normal protein and glucose with matched oligoclonal bands in serum and CSF. Standard CSF neuroviral PCR panel and PCR for SARS-CoV-2 were negative.

Extensive antibody screening including for N-methyl-D-aspartate receptor (NMDA-R), glutamic acid decarboxylase (GAD), contactin-associated proteinlike 2 (CASPR2), leucine-rich, glioma inactivated 1 (LGI1) antibodies, ganglioside antibodies and a panel of antineuronal antibodies in serum and CSF were negative. Antiglycine receptor and anti dipeptidyl-peptidase-like protein 6 (DPPX) antibodies were negative.

CT of the chest, abdomen and pelvis confirmed lung changes of COVID-19 but no evidence of malignancy. Electroencephalogram was within normal limits with no cortical correlate to the myoclonus. Fluorodeoxyglucose positron emission tomography (FDG-PET) was consistent with pneumonitis with no other pathological uptake seen.

Levetiracetam $750 \mathrm{mg}$ two times a day and clonazepam $0.25 \mathrm{mg}$ two times a day were commenced and over the following week there was partial improvement in myoclonus and hyperekplexia, but no significant improvement in cognition, mobility or eye movement abnormalities. Repeat testing for SARS-CoV-2 on day 5 and day 7 was negative and no symptoms of active COVID-19 infection were seen during her admission.

The patient was discussed in our multidisciplinary teleconferencing meeting and as the most likely cause of her neurological syndrome was felt to be a postinfectious immune-mediated encephalitis a decision was made to treat with corticosteroids.

Fourteen days following onset of neurological symptoms (21 days following onset of COVID-19 symptoms), she was commenced on intravenous methylprednisolone $1 \mathrm{~g}$ per day for three consecutive days. This was followed by oral prednisolone $60 \mathrm{mg}(1 \mathrm{mg} / \mathrm{kg})$, with a plan to wean this to cessation over a period of 4-6 months.

Following initiation of corticosteroids there was slow progressive improvement in her neurological symptoms. On discharge from hospital (10 days after steroids), she was able to mobilise with one stick and cognition had returned to her previous baseline. There was ongoing fine myoclonus however this was continuing to improve.

\section{CONCLUSION}

This case expands on emerging literature describing postinfectious immunemediated neurological sequelae affecting the central nervous system in patients with COVID-19. While causation is difficult to prove, the temporal profile of symptoms developing 1 week following COVID-19 clearly implicates viral infection as a contributing factor.

While we could not identify evidence of inflammation on neuroimaging, this is not uncommon in other forms of autoimmune encephalitis, ${ }^{3}$ and her clinical presentation was strongly suggestive of a postinfectious 
cortical but predominantly brainstem syndrome.

Although it is unclear whether treatment with steroids was a contributing factor to her improvement or whether this was part of the natural history of her illness, this case highlights a possible role for steroids in patients where a postCOVID-19 autoimmune encephalitis is suspected, after exclusion of appropriate mimics. Resolution of fever and respiratory symptoms together with repeated negative SARS-CoV-2 PCR and normal lymphocyte count reassured us she was no longer actively infected and made us more comfortable in starting corticosteroids rather than considering other immunomodulatory options like intravenous immunoglobulin or plasma exchange.

\section{Anthony Khoo $\odot$,' Benjamin McLoughlin, ${ }^{1}$ Sanjay Cheema $10,{ }^{1}$ Rimona S Weil, ${ }^{1,2}$ Christian Lambert, ${ }^{1}$ Hadi Manji, Michael S Zandi, ${ }^{1,3}$ Jasper M Morrow ${ }^{1}$}

1 Department of Neurology, University College London Hospitals NHS Foundation Trust National Hospital for Neurology and Neurosurgery, Queen Square, UK
${ }^{2}$ Dementia Research Centre, University College London, London, UK

${ }^{3}$ Queen Square Institute of Neurology, University College London, London, UK

Correspondence to Dr Anthony Khoo, Department of Neurology, University College London Hospitals NHS Foundation Trust National Hospital for Neurology and Neurosurgery, Queen Square, London WC 1N 3BG, UK anthony.khoo@sa.gov.au

Twitter Michael S Zandi @michael_zandi

Contributors All authors were involved in the clinical care of the patient. AK, BM and SC wrote the first draft of the manuscript. AK, CL and RSW provided clinical videos. All authors edited, critically revised and approved the final manuscript for submission.

Funding The authors have not declared a specific grant for this research from any funding agency in the public, commercial or not-for-profit sectors.

Competing interests None declared.

Patient consent for publication Next of kin consent obtained.

Provenance and peer review Not commissioned; externally peer reviewed.

(C) Author(s) (or their employer(s)) 2020. No commercial re-use. See rights and permissions. Published by BMJ.

- Additional material is published online only. To view please visit the journal online (http://dx.doi.org/ 10.1136/jnnp-2020-323816)
Check for updates

To cite Khoo A, McLoughlin B, Cheema S, et al. J Neurol Neurosurg Psychiatry 2020;91:1013-1014.

Received 12 May 2020

Revised 5 June 2020

Accepted 8 June 2020

Published Online First 7 July 2020

J Neurol Neurosurg Psychiatry 2020;91:1013-1014. doi:10.1136/jnnp-2020-323816

\section{ORCID iDs}

Anthony Khoo http://orcid.org/0000-0003-3493-5202

Sanjay Cheema http://orcid.org/0000-0002-5438-6549

\section{REFERENCES}

1 Zhao H, Shen D, Zhou H, et al. Guillain-Barré syndrome associated with SARS-CoV-2 infection: causality or coincidence? Lancet Neurol 2020;19:383-4.

2 Rábano-Suárez P, Bermejo-Guerrero L, Méndez-Guerrero A, et al. Generalized myoclonus in COVID-19. Neurology 2020;21:1-3.

3 Irani SR, Bera K, Waters P, et al. N-Methyl-D-Aspartate antibody encephalitis: temporal progression of clinical and paraclinical observations in a predominantly non-paraneoplastic disorder of both sexes. Brain 2010;133:1655-67. 